argue in favor of aggressive treatment of uncontrolled recurrent seizures, but the use of antiepileptic drugs to prevent epilepsy in children having single or infrequent febrile or afebrile seizures remains controversial. Most authorities do not recommend long-term continuous AEDs for prevention of febrile seizures. Each child is an individual, the decision to start AEDs depending on the cause, severity, associated psycho-neurological findings, and likely prognosis of the seizure disorder, and the potential toxicity of the medication. (see Progress in Pediatric Neurology I, 1991;pp18-21). In adults, early treatment of a single seizure has been shown to prevent recurrence. (see Progress in Pediatric Neurology III, 1997;pp112-113).

\title{
MECHANISMS OF PHOTOSENSITIVE EPILEPSIES
}

Intermittent photic stimulation was performed in 21 photosensitive epileptic patients and the relation of the photoparoxysmal response (PPR) to the epilepsy syndrome was determined in a study at the National Epilepsy Center, Shizuoka Higashi Hospital, Shizuoka, Japan. The PPR was dependent on the quantity-of-light in 4 of 6 patients with photosensitive myoclonic epilepsy in infancy, and in 2 of 7 with symptomatic generalized epilepsy. Wavelength dependent PPR was elicited in 5 of 8 patients with photosensitive localizationrelated epilepsy, and in 4 of 7 with symptomatic generalized epilepsy. The type of pathophysiologic mechanism for eliciting PPRs by low-luminance photic stimulation was correlated with the epilepsy syndrome classification. (Takahashi Y, Fujiwara T, Yagi K, Seino M. Photosensitive epilepsies and pathophysiologic mechanisms of the photoparoxysmal response. Neurology Sept 1999;53:926-932). (Reprints: Dr Y Takahashi, Department of Pediatrics, Gifu University School of Medicine, 40 Tsukasa-machi, Gifu 500-8076, Japan).

COMMENT. The high frequency of photosensitivity in patients with severe myoclonic epilepsy of infancy might be related to the quantity-of-lightdependent (QLD) mechanism. In the above study, patients with QLD-PPRs have PPRs at frequencies from 6 to $33 \mathrm{~Hz}$, whereas patients with wavelength-dependent (WLD) PPRs show responses at a narrower range $(12-20 \mathrm{~Hz})$. This suggests a higher level of photosensitivity in patients with QLD than WLD PPRs. Some photosensitive patients are thought to undergo an evolution and a change in mechanism with age.

\section{$\underline{\text { SLEEP DISORDERS }}$}

\section{SLEEP DISTURBANCES AND ATTENTION DEFICIT DISORDER}

The relationship of sleep disorders, attention deficit hyperactivity disorder (ADHD), comorbidity associated with ADHD, and treatment with stimulant medications was determined using 2 sleep questionnaires completed by the parents in a study of children aged 6 to 12 years treated at the Hospital for Sick Children, Toronto, Canada. Sleep problems and factors associated with sleep difficulties were compared in ADHD unmedicated patients $(n=79)$, medicated ADHD $(\mathrm{n}=22)$, and 2 control groups. The percentage of subjects with 1 or more sleep problems was highest in the medicated ADHD group $(95.5 \%)$ and unmedicated ADHD group (86.1\%); it was $82.9 \%$ in the clinical comparison group, and $55.5 \%$ in the nonclinical comparison group. Three sleep problems were evident by factor analysis: dysomnias (difficulty getting up, going to bed, and/or falling asleep; parasomnias (sleep walking, night waking, sleep terrors); and sleep related involuntary movements. 
Dysomnias were related to comorbid oppositional defiant disorder and stimulant medication, but not to ADHD. Parasomnias were similar in clinical and nonclinical controls. Sleep-related involuntary movements were increased in frequency in children with the combined subtype of ADHD and in those with anxiety. Although children with $\mathrm{ADHD}$ had more sleep problems than normal children, they did not differ from those in the control group referred for a clinical assessment. (Corkum P, Moldofsky H, Hogg-Johnson S, Humphries T, Tannock R. Sleep problems in children with attention-deficit/hyperactivity disorder: Impact of subtype, comorbidity, and stimulant medication. I Am Acad Child Adolesc Psychiatry October 1999;38:1285-1293). (Respond: Dr Tannock, Department of Psychiatry Research, Hospital for Sick Children, 555 University Avenue, Toronto, Ontario, Canada M5G 1X8).

COMMENT. Children with ADHD, in comparison with normal children, experience more sleep problems evidenced by dyssomnias and involuntary movements, but the frequency of parasomnias is similar to that in normally developing peers. Except for the association of sleep-related involuntary movements and the combined subtvpe of ADHD, sleep problems in ADHD children do not differ from those in clinical comparison groups without ADHD. Dysomnias in children with ADHD occur especially in those with comorbid ODD or in children taking stimulant medication. Involuntary movements during sleep are strongly associated with anxiety, especially separation anxiety.

The International Classification of Children's Sleep Disorders, a more comprehensive and current listing than the DSM, includes 1) dyssomnias, or primary sleep disorders (eg. obstructive sleep apnea, narcolepsy, periodic limb movements, inappropriate environment and parenting, circadian sleep-wake rhythm disorders); 2) parasomnias (eg. sleepwalking and sleep terrors - arousal from deep NREM sleep, headbanging - sleep-wake transition disorders, nightmares - with REM sleep; and 3) sleep disorders associated with neuropsychiatric or other medical disorders (eg. depression, epilepsy, asthma). (Stores G. Children's sleep disorders: modern approaches, developmental effects, and children at special risk. Dev Med Child Neurol Aug 1999;41:568-573). The effects of sleep disorders on cognition, emotional state and behavior are discussed, and the need for a greater professional awareness of the problem is stressed.

\section{SLEEP DISORDERS IN BLIND CHILDREN}

The frequency and type of sleep disorders in 77 blind children, from 3 to 18 years of age, were compared with 79 matched controls, using a 42-item questionnaire in a study at the Stanford University Sleep Disorders Center, Stanford, CA, and the Laboratoire du Sommeil, Centre Hospitalier Universitaire de l'Hotel-Dieu a Paris, France. Questions were derived from the DSM-IV and the ICSD90, and were answered by the child alone or with parental help. None had mental retardation nor any chronic illness aside from blindness. Only $8 \%$ had a circadian rhythm disorder (dyschronosis). Blindness occurred in perinatal life in $75 \%$ and after 1 year of life in $25 \%$.

Mean total sleep time was similar in both groups; it was $526+/-61$ minutes on weekdays and $600 \mathrm{~min}$ on weekends. A significantly greater proportion of blind children (17\%) slept less than 7 hours per night on weekdays compared to controls (2.6\%), and blind subjects had more sleep complaints (difficulty in getting to sleep, awakening too early, poor quality sleep, sleepwalking, bruxism). Daily episodic involuntary sleepiness occurred in 13\% of blind children compared to $1.3 \%$ of controls. Age of onset of blindness and presence or absence of light 